\title{
" EFFECT OF WINTER CHERRY EXTRACT (WITHAFERIN-A) ON HEAD AND NECK SQUAMOUS CELL CARCINOMA CELL LINE" IN VITRO STUDY
}

\author{
Enjy Abdelarzek Abd Elkalik Bassim*, Dr. Mohammed Hossam \\ Eldin Elmallahi** and Dr. Nermeen Sami Afifi***
}

\section{Introduction:}

Oral cancer is the sixth most common cancer worldwide (Dhanuthai, et al., 2018). More than $90 \%$ of all oral cancers are squamous cell carcinoma (SCC) (Nayanar, 2019). The use of tobacco and betel quid, heavy drinking of alcoholic beverages and a diet low in fresh fruits and vegetables are well known risk factors for oral squamous cell carcinoma (Feller and Lemmer, 2012).

Naturally occurring agents are known to have several drug extracts that have anticancer effect (Gordaliza , 2007), (Merlin, Parthasarathy and Santhoshkumar, 2010), (Safe and_Kasiappan, 2016). Many studies showed the effectiveness of Withaferin -A as an anticancer therapy for different types of cancers (McKenna, et al., 2015), pancreatic cancer (Cai, et al., 2014).

\section{Review of Literature:}

Oral cancer is considered to be within the top 10 cancers in the world (Rivera, 2015). Among oral cancers, squamous cell carcinoma represents about (90-95\%) of oral cancers. Some conditions are related to the occurance of OSCC like PlummerVinson syndrome, Xeroderma pigmentosa, Fanconi anemia, dyskeratosis congenita, discoid lupus erythematosus, oral submucous fibrosis, leukoplakia and lichen planus ( Villa and Sonis, 2018). Most commonly affected sites are the ventral surface of the tongue and floor of the mouth, and this may be due to the thin non-keratinized epithelium lining these sites and the ease of diffusion of different carcinogens

* B.D.S Faculty of Dentistry Ain Shams University

** Professor of Oral Pathology Ain Shams University President of Nahda University

*** Lecturer of Oral Pathology Ain Shams University 
through this thin lining (Britta, et al., 2018). OSCC may take various clinical forms, it may be look-like leukoplakia, erythroplakia or erythroleukoplakia. It may also be an exophytic mass which ulcerate subsequently or take the shape of an ulcer from the beginning (Bagan, et al., 2010).

There are different modalities for the treatment of OSCC, including excision/ resection, radio-therapy, systemic cytotoxic chemotherapy and blocking of epithelial growth factor receptor (EGF-R), or a combination of these, and using them either concurrently or in an orderly sequence. Surgery is the first line treatment of small and accessible OSCCs, but advanced-stage OSCC is treated by a combined treatment program of surgery, chemotherapy and radiotherapy (Shah and Gil, 2009), (Braakhuis, et al., 2010), (Rapidis, et al., 2009), (Mazeron, et al., 2009) and (Ribeiro, et al., 2016).

Chemotherapeutics were reported to be linked with wide variety of side effects, such as, fatigue, nausea, vomiting, malaise, diarrhea, mucositis, pain, rashes, infections, headaches and several other problems. (Loprinzi, et al.,2007), (Brewer, et al., 2016).

Plants are natural factories for synthesizing structurally different chemical compounds, such as steroids, terpenoids, Davonoids, alkaloids, etc. Many of these secondary metabolites are biologically active, interacting directly or indirectly with different cellular components, especially proteins and lipids, reversing the altered functions of cancer cells (Surh, 2003).

One of these phytochemicals is the winter cherry extract Withaferin-A (W-A). It is derived from the medicinal plant Withania Somnifera (L.) Dunal (Family Solanaceae). This herbal plant also known as Ashwaganda, Indian winter cherry or Indian ginseng (Vyas and Singh, 2014).

Beside its anti inflammatory and anti oxidant proparties, WA has been shown to suppress cell proliferation either in a variety of cancer cells in culture and in mouse xenograft tumor models (Vyas and Singh, 2014). W-A showed cytotoxic effect against a wide array of cancer cells. (Mandal, et al., 2008). (Samadi, et al., 2010) reported the apoptotic effect of $\mathrm{W}-\mathrm{A}$ in human head and neck squamous cell carcinoma (HNSCC) cells.

Cell death, particularly apoptosis, is probably one of the most widely-studied subjects among cell biologists. Understanding apoptosisis not important in the disease pathogenesis only but also it benefits in the detection of treatment of diseases. Understanding of apoptosis and mechanisms of action of it will assist in the evolution of new treatment options of cancer as the problem can arise in any one step along the way of apoptosis (Su,.et al., 2015).

There are three pathways the activation of caspases has been accommplished. The two commonly known initiation pathways are the intrinsic (or mitochondrial) and extrinsic (or death receptor) pathways of apoptosis. Both pathways lead to finally a common pathway or the execution phase of apoptosis. A third less well-known initiation pathway is the intrinsic endoplasmic reticulum pathway.

Defects in apoptosis mechanisms have major roles in tumor pathogenesis, allowing tumour cells to survive over intended lifespans, so providing protection for neoplastic cells from hypoxic conditions and oxidative stresses allowing the tumor mass to expand, giving time for cellular genetic alterations with subsequent deregulated cellular proliferation and interference with cellular differentiation, promotion of angiogenesis and the increase of tumor invasiveness during the progression of the tumor mass (Reed, et al., 1998).

Successful eradication of tumoureous cells by nonsurgical means is approached via induction of apoptosis. So, all the cancer drug designers try either to activate the inactivated apoptotic mechanism or rectify a defective one.

\section{Aims of the Study}

The present study aimed to:

1- investigate the possible cytotoxic effect of winter cherry extract (Withaferin-A) on head 
and neck squamous cell carcinoma cell line.

2- evaluate the possible cytological changes of winter cherry extract (Withaferin-A) treated head and neck squamous cell carcinoma cell lines.

\section{Material and Methods:}

\section{Material}

Human tongue squamous cell carcinoma cell line (SCC 25) having the morphology of epithelial cells, was supplied from the Cell Culture Department - VACSERA, EGYPT. Withaferin-A the cytotoxic drug used in this study, was purchased from Sigma AldrichUSA. In-vitro viability assay kit (Sigma AldrichUSA), MTT (3, 4, 5-dimethylthiazol-2, 5-diphenyltetrazolium bromide) $15 \mathrm{mg} / \mathrm{vial}$ in serum and MTT solubilization solution $10 \%$ Triton X-100 plus $0.1 \mathrm{~N} \mathrm{HCL}$ in anhydrous isopropanol, $125 \mathrm{ml}$ was used for viability testing.

\section{Methods}

Cell culture was performed in The Cell Culture Unit - VACSERA, Egypt, then checked under inverted light microscope. Cells were placed (cells density $1.2-1.8 \times 10,000$ cells/ well) in a volume of $100 \mu \mathrm{l}$ complete growth medium +100 ul of Withaferin-A per well in a 96-well plates (figure: 6) for 24 hours to perform MTT assay. Cells were treated with serial doses $(0.39,1.56,6.25,25$ and 100 uM) of Withaferin-A to be tested. Non-treated control wells were ethanol treated. Plates were incubated for 24 and 48 hours at $37^{\circ} \mathrm{C}$. . After the incubation period, cultures were removed from the incubator and the resulting purple formazan crystals were dissolved by adding an amount of MTT solubilization solution [M8910] equal to the original culture medium volume. Absorbance was measured using ROBONIK P2000 Elisa Reader at a wavelength of $450 \mathrm{~nm}$. The percentage of viable cells was calculated according to the following equation (Boo et al., 2018)

The data recorded from the MTT assay are used to calculate the IC50 of WA at both duration. For further investigations certain doses were choosed at both durations, guided by previous studies and determined according to the IC50 values.

Hematoxylin and Eosin Staining was performed in Oral pathology lab, Faculty of Dentistry, Ain-Shams University for cytological examination and further investigations. Photomicrography Cytological Examination and Nuclear Morphometric Analysis was performed in the Precision Measurement Unit, Oral Pathology Department, Faculty of Dentistry, Ain Shams University. The photomicrographed fields were analysed using Image J, 1.41a, (NIH, USA) image analysis software for Nuclear Morphometric Analysis.

\section{Results:}

\section{MTT Assay Results:}

MTT assay was performed to determine the cytotoxic effect of serial doses of W-A for 24 and $48 \mathrm{hrs}$ and to calculate the IC50. Data recorded revealed that the cytotoxicity of W-A to SCC 25 cell line was relatively proportional to different doses of $\mathrm{W}-\mathrm{A}$ at both durations. There was progressive decrease in the mean viability percentage in W-A treated SCC 25 cells with increasing the doses of the drug for both durations.

\section{Cytological Examination Results:}

Cytological examination was performed after incubation of SCC 25 cells with the different doses of W-A at different durations ( $24 \mathrm{hrs}, 48 \mathrm{hrs}$ ). The cytological examination of the different groups revealed that the amount and severity of apoptosis was affected by the dose of the $\mathrm{W}-\mathrm{A}$ as well as the duration of exposure to the drug. Increasing the dose and duration of treatment was found to be associated with increase in the amount of cells showing criteria of apoptosis as well as the severity of apoptotic signs compared to control cells at both durations.

\section{Nuclear Morphometric Analysis Results:}

Nuclear Area Factor (NAF): 
The data recorded revealed that after $24 \mathrm{hrs}$ of treatment of SCC 25 cells with different doses of $\mathrm{W}-\mathrm{A}$, there was progressive decrease in the mean values of NAF when compared to that of control cells with the lowest value 2545.58 in W3T1 group and the highest value 4080.56 in W1T1 (Table:1) and (Figure: 1).

Table: (1) The mean values and standard deviation of NAF of SCC25 cells treated with different doses of $\mathrm{W}-\mathrm{A}$ at $24 \mathrm{hrs}$

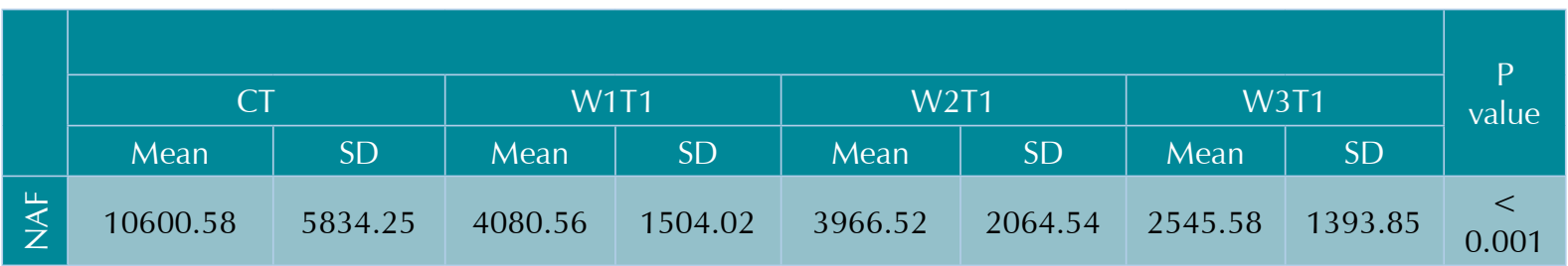

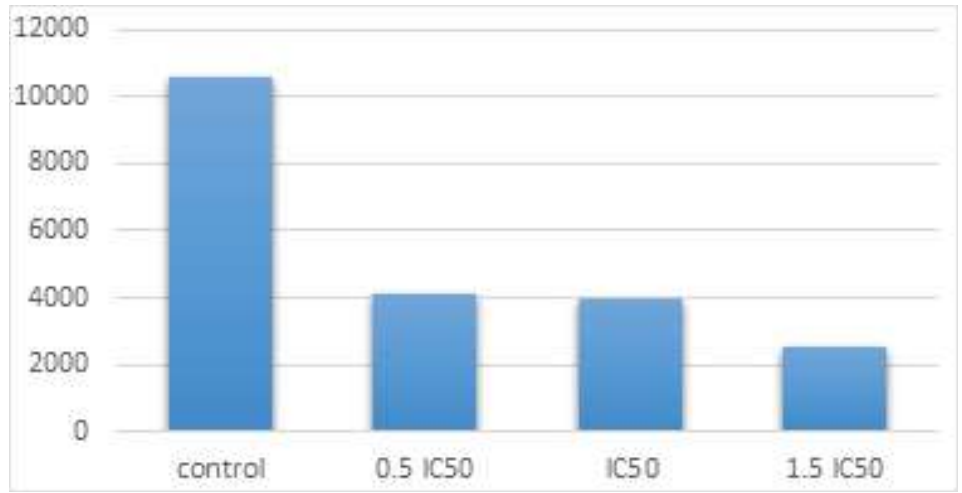

Figure: (1) A bar chart representing the mean values of NAF of SCC25 cells treated with different doses of W-A for 24 hrs.

Kruskal-Wallis test revealed that there was statistically high significant difference for the NAF in different groups of W-A treated SCC25 cells at 24 hours ( $p$ value $=0.001$ ) (Table: 1 ).

Increasing the duration to 48hrs treating the groups of SCC 25 cells named CT2, W1T2, W2T2 and $\mathrm{W} 3 \mathrm{~T} 2$, revealed a progressive decrease in the mean values of NAF with increasing the dose of W-A as showen in (Table: 2) that display the values of NAF and their standard deviation.

The lowest value of NAF mean was observed in W3T2 group 1437.84, and the highest value was 2741.08 at W1T2 group. (Table: 2), (Figure: 2).

Table: (2) The mean values of NAF and standard deviation of different groups of W-A treated SCC25 cells at 48 hours post treatment.

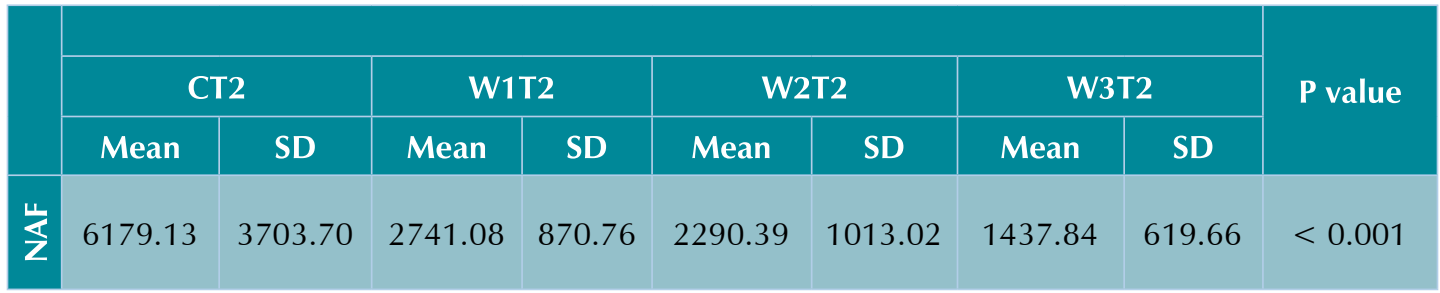




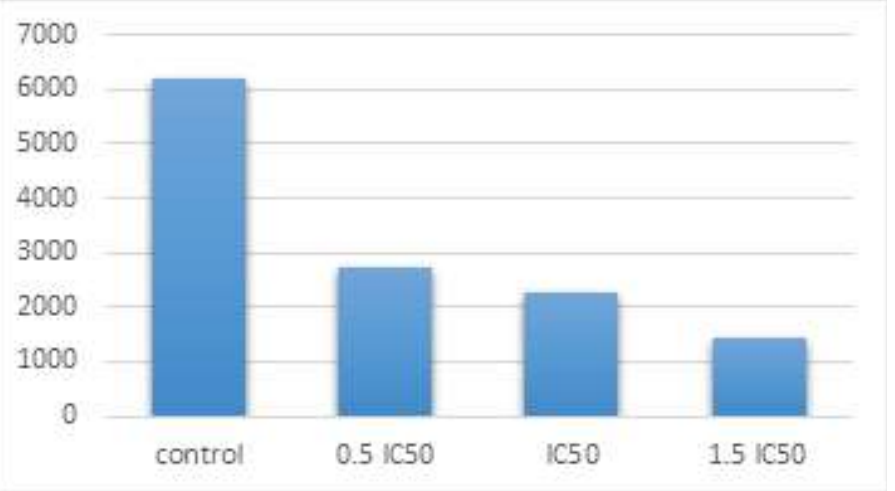

Figure: (2) A bar chart representing the mean values of NAF of SCC 25 cells treated with different doses of $W$-A for 48 hrs.

Kruskal-Wallis test revealed that there was high statistically significant difference between the mean NAF values in different groups of W-A treated SCC25 cells at 48 hours ( $p$ value $<0.001$ ). (Table: 2).

\section{Discussion:}

HNSCCs have a yearly incidence of 600,000 cases worldwide, with 40-50\% mortality (Ferlay et al., 2015). Oral cancer is estimated to be the sixth most common cancer worldwide, and more than $90 \%$ of oral cancers are OSCCs. (Elnaggar et al., 2017). Chemotherapeutic drugs are associated with a wide spectrum of hematologic toxicities that include anemia, leukopenia, neutropenia and thrombocytopenia (Buglione et al., 2016).

Withania Somnifera, commonly known as Ashwagandha belong to family Solanaceae, it is one of the greatly important ancient medicinal plant in Ayurvedic medicine (Kumar et al., 2013). Withaferin-A (W-A) is one of its most important bioactive compounds. The Anticancer activity of W-A has been reported against wide range of different cancers such as, prostate (Srinivasan et al., 2007); leukemia (Oh et al., 2008) and (Mandal et al., 2008).

The present study aimed to investigate the possible cytotoxic effect of W-A on one of SCC cell lines (SCC 25), evaluate the cytological and nuclear morphometric changes that might occur in W-A treated cells in comparison to untreated control cells.

Increasing the dose of $\mathrm{W}$-A is highly toxic to the viable cultured cells, an observation in accordance with that reported in the study of (Kim et al.,2017) on human gastric carcinoma cell line. W-A dose needed to kill half number of the cells after 48hrs (IC50; 9.93 uM) was much more lower than that needed to kill the same amount of cells after 24hrs (IC50; 42.22 uM) which confirm the effect of the duration of the treatment on the used doses of the drug and allowing the use of lower doses with lower side effects- if present- by increasing the treatment duration.

W-A treated SCC 25 cells were evaluated cytologically to compare between cancer cells before and after W-A administration to predict the effect of the drug on induction of apoptosis. Cytological examination of W-A treated SCC 25 cells stained with hematoxylin and eosin at the power of 1000x original magnification (oil immersion) revealed the presence of morphological criteria of apoptosis in W-A treated cells which were nearly absent in control cells.

In this study, the progressive decrease in the mean values of nuclear circularity and nuclear surface area from control group to $\mathrm{W}$-A treated groups in both durations, resulting in dramatic statistically significant reduction of NAF from 10600.58 in control cells to 2545.58 in SCC 25 cells treated with 1.5 IC50 of W-A at 24 hrs, and from 6179.13 to 1437.84 after $48 \mathrm{hrs}$. 


\section{Conclusions:}

W-A has a cytotoxic effect on squamous cell carcinoma cell line in a dose and time dependent manner.

The estimated optimum W-A dose that induced maximum apoptotic changes on squamous cell carcinoma cell line in this study is (1.5 IC50) after $48 \mathrm{~h}$ of treatment.

As the duration of treatment increased, the doses required to induce maximum apoptotic changes and minimum necrotic changes decreased.

\section{Recommendations:}

Further studies are recommended using other concentrations of W-A at different durations.

Further studies to determine the effect of W-A on other types of cancer cell lines as well as normal cell lines (non-cancerous) are needed.

\section{Referances:}

Boo H., Park J., Lee M., Kwon S. and Kim H.: In-vitro cytotoxicity against human cancer cell and 3T3-L1 cell, total polyphenol content and DPPH radical scavenging of codonopsis lanceolata according to the concentration of ethanol solvent. Korean J. Plant Res.; 31: 195201, 2018.

Bottone M., Santin G., Aredia $_{\text {F., Pellicciari }}$ C. Bernocchi G. and Scovassi A.: Morphological features of organelles during apoptosis: An Overview. Cel. J.; 2: 2013 ,305-294.

Brewer J., Morrison G., Dolan M. and Fleming G.: Chemotherapy-induced peripheral neuropathy: Current status and progress. Gyne. Oncol. J.; 140: 176-183, 2016.

Buglione M., Cavagnini R., Di Rosario F., Sottocornola L. and Maddalo M.: Oral toxicity management in head and neck cancer patients treated with chemotherapy and radiation: dental pathologies and osteoradionecrosis (Part 1) literature review and consensus statement. Crit. Rev. Oncol. Hematol.; 97: 131-142, 2016.
Cai Y., Sheng Z., Chen Y. and Bai C.: Effect of Withaferin A on A549 cellular proliferation and apoptosis in non-small cell lung cancer. Asi. Paci. J. of Canc. Prev.; 15: 1711-1714, 2014.

Dhanuthai K., Rojanawatsirivej S., Thosaporn W., Kintarak S., Subarnbhesaj A., Darling M., et al.,: Oral cancer: A multicenter study. Med. Oral Pathol. Oral Cir. Bucal.; 23: 23-29, 2018.

Elnaggar A., Chan J., Grandis J., Takata T. and Slootweg P.: WHO classification of head and neck tumours. (edition 4), Lyo.; 110, 2017.

Feller L. and Lemmer J.: Oral squamous cell carcinoma: Epidemiology, clinical presentation and treatment. J. of Canc. Ther.; 3: 263-268, 2012.

Ferlay J., Soerjomataram I., Dikshit R., Eser S., Mathers C., Rebelo M., et al.: Cancer incidence and mortality worldwide: sources, methods and major patterns in GLOBOCAN 2012. Int. J. Cancer; 136: 359-386, 2015.

Gordaliza M.:Natural products as leads to anticancer drugs. Clini.Trans. Onco.; 9: 767776, 2007.

Kim G., Kim T., Hwang E., Chang K., Hong J. and Park J.: Withaferin-A inhibits the proliferation of gastric cancer cells by inducing $\mathrm{G} 2 / \mathrm{M}$ cell cycle arrest and apoptosis. Onco. Let.; 416-422, 2017.

Kumar V., Abbas A., Fausto N. and Aster J.: Robins and Cotran: pathologic basis of disease. edition 8. Phila. Sau. Else.; 25-32, 2010.

Malik F., Kumar A., Bhushan S., Khan S. and Bhatia A., Suri K., et al.: Reactive oxygen species generation and mitochondrial dysfunction in the apoptotic cell death of human myeloid leukemia HA-60 cells by a dietary compound withaferin-A with concomitant protection by $\mathrm{N}$-acetyl cysteine. Apopt.; 12: 2115-2133, 2007.

Mandal C., Dutta A., Mallick A., Chandra S., Misra L.and Sangwan R.: Withaferin-A induces apoptosis by activating p38 mitogenactivated protein kinase signaling cascade in leukemic cells of lymphoid and myeloid origin 
through mitochondrial death cascade. Apopt.; 13: 1450-1464, 2008.

Mazeron R., Tao Y., Lusinchi A. and Bourhis J.: "Current concepts of management in radiotherapy for head and neck squamous cell cancer". Or. Onco.; 45: 402-408, 2009.

McKenna M., Gachuki B., Alhakeem S., Oben K., Rangnekar V., Gupta R., et al.: Anti-cancer activity of Withaferin-A in B-cell lymphoma. Canc. Bio. \& Ther.; 7:1088-1098, 2015.

Merlin N., Parthasarathy V. and Santhoshkumar T.: Induction of apoptosis in human breast cancer cell line MCF-7 by phytochemicals from GmelinaAsiatica. Afri. J. of Biotech.; 9: 4451-4454, 2010.

Murata S., Mochizuki K., Nakazawa T., Kondo T., Nakamura N. and Yamashita H.: Morphological abstraction of thyroid tumor cell nuclei using morphometry with factor analysis. Microsc. Res. Tech.; 61: 457-462, 2003.

Nayanar S., Tripathy J., Duraisamy K., and Babu S.,: Prognostic efficiency of clinicopathologic scoring to predict cervical lymph node metastasis in oral squamous cell carcinoma. J. Or. Maxillofac. Pathol.; 23: 3642, 2019.

Reed J., Jurgensmeier J., and Matsuyama S.: "Bcl2 family proteins and mitochondria". Biochimicaet. Biophysica. Acta.; 1366: 127137,1998.

Ribeiro P., Barroso L., Marques F., Melo J. and Carreira I.: Early detection and personalized treatment in oral cancer: the impact of omics approaches. Mol. Cytogen. J.; 9, 2016.

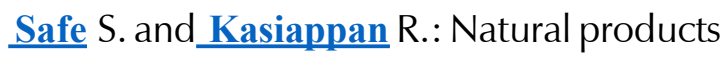
as mechanism-based anticancer agents: Sp transcription factors as targets. Wil. On. Lib. 30: 1723-1732, 2016.

Samadi A., Mukerji R., Shah A., Timmermann B., Cohen M.: A novel RET inhibitor with potent efficacy against medullary thyroid cancer in-vivo. Surg.; 148: 12281236, 2010.

Srinivasan S., Ranga R., Burikhanov R., Han S., Chendil D.: Par4-dependent apoptosis by the dietary compound withaferin-A in prostate cancer cells. Canc. Res.; 67: 246-253, 2007.

Su Z., Yang Z., Xu Y., Chen Y. and Yu Q.: Apoptosis, autophagy, necroptosis, and cancer metastasis. Mole. Canc.; 14: 48, 2015.

Surh Y.: Cancer chemo-prevention with dietary phytochemicals. Nat. Rev. Canc.; 3: 768-780, 2003.

Villa A. and Sonis S.: Oral leukoplakia remains a challenging condition. J. Prof. Scu. Memo.; 24: 179-183, 2018.

Vyas A., and Singh S.: Molecular targets and mechanisms of cancer prevention and treatment by withaferin-A, a naturally occurring steroidal lactone. AAP. J.; 16: 1-10, 2014. 
